

\title{
INTERACCIONES Y CONTRIBUCIONES. FORMA DE PARTICIPACIÓN DE ESTUDIANTES DE QUINTO GRADO EN AMBIENTES DE MODELACIÓN MATEMÁTICA
}

INTERACTIONS AND CONTRIBUTIONS. WAY OF PARTICIPATION OF FIFTH-GRADE

STUDENTS IN MATHEMATICAL MODELLING ENVIRONMENTS

\author{
Volumen 16, Número 3 \\ Setiembre-Diciembre
}

pp. 1-27

DOI: http://dx.doi.org/10.15517/aie.v16i3.26084

Mónica Marcela Parra-Zapata

Jhony Alexander Villa-Ochoa

Revista indizada en REDALYC, $\underline{\text { SCIELO }}$

Revista distribuida en las bases de datos:

LATINDEX, DOAJ, E-REVIST@S, IRESIE, CLASE, DIALNET, SHERPA/ROMEO, QUALIS,

Revista registrada en los directorios:

ULRICH'S, REDIE, RINACE, OEI, MAESTROTECA, PREAL, $\underline{\text { CLACSO }}$ 


\title{
INTERACCIONES Y CONTRIBUCIONES. FORMA DE PARTICIPACIÓN DE ESTUDIANTES DE QUINTO GRADO EN AMBIENTES DE MODELACIÓN MATEMÁTICA \\ INTERACTIONS AND CONTRIBUTIONS. WAY OF PARTICIPATION OF FIFTH-GRADE STUDENTS IN MATHEMATICAL MODELLING ENVIRONMENTS
}

\author{
Mónica Marcela Parra-Zapata ${ }^{1}$ \\ Jhony Alexander Villa-Ochoa ${ }^{2}$
}

\begin{abstract}
Resumen: La literatura internacional llama la atención sobre la importancia de promover espacios de participación en ambientes de aprendizaje de la matemática escolar; sin embargo la cantidad de investigaciones que se ocupe de estudiarla en ambientes de modelación en educación primaria es insuficiente. En este sentido, se desarrolló una investigación en la cual se indagó las maneras cómo un grupo de estudiantes participó en ambientes que incluían algunas características de la perspectiva socio-crítica de la modelación matemática, entre ellas, la posibilidad de negociación, de discusión y el cuestionamiento del rol de las matemáticas en los fenómenos y en la sociedad. La investigación fue cualitativa y en ella participaron 27 estudiantes de quinto grado (9-11 años) de una institución educativa de la Ciudad de Medellín. Para analizar la participación de los estudiantes se utilizaron entrevistas, observación participante y documentos. Se centró la atención en las manifestaciones orales y escritas, las acciones y las explicaciones de los estudiantes en dos (2) episodios ocurridos en ambientes de modelación matemática. Los resultados permitieron identificar que cuando los estudiantes se involucran en ambientes que promueven su participación, se comprometen con la descripción e interpretación de las situaciones a estudiar, la matematización de relaciones propias de la situación y la interpretación y el análisis de las soluciones propuestas. En consecuencia, se logró analizar y caracterizar las interacciones y las contribuciones como una forma de participación. Los resultados ofrecen una comprensión de la participación de los estudiantes en ambientes de modelación matemática, y por tanto, algunas nociones y aportes teóricos en Educación Matemática.
\end{abstract}

Palabras clave: EDUCACIÓN PRIMARIA, AMBIENTES DE MODELACIÓN, PERSPECTIVA SOCIO-CRÍTICA, PARTICIPACIÓN, MODELACIÓN MATEMÁTICA,

\begin{abstract}
International literature attracts attention to the importance of promoting opportunities for participation in learning environments of school mathematics; however, the amount of research that deals with modeling study in environments in primary education are insufficient. In this sense it was carried out a research that inquiries about the ways how a group of students participated in environments which included some features of the socio-critical perspective of mathematical modelling, including negotiation possibilities, discussion possibilities and questioning the role of mathematics on the phenomena and society. The research was qualitative and it involved 27 fifth-grade students (9-11 years old) of an educational institution in the city of Medellin. To analyze the participation of the study, the instruments to collect data were interviews, participant observation and documents. The study focused the attention on oral and written statements, actions and explanations of the students in two (2) episodes in mathematical modelling environments. The results allowed to identify when students engage in environments that promote their participation, agree with the description and interpretation of the situations studied, the mathematization of own relationships of the situation and the interpretation and analysis of the proposed solutions. Consequently, it was possible to analyze and characterize the interactions and contributions as a form of participation. The results provide an understanding of the participation of the students in mathematical modeling environments, therefore, some notions and theoretical contributions in Mathematics Education.
\end{abstract}

Keywords: PRIMARY EDUCATION, MODELLING ENVIRONMENTS, SOCIO-CRITICAL PERSPECTIVE, PARTICIPATION, MATHEMATICAL MODELLING.

\footnotetext{
1 Profesora, Magíster en Educación. Línea Educación Matemática. Universidad de Antioquia, Colombia. Dirección electrónica: monica.parra@udea.edu.co

2 Profesor asociado. Doctor en Educación. Línea Educación Matemática. Universidad de Antioquia, Colombia. Dirección electrónica: ihony.villa@udea.edu.co
}

Artículo recibido: 18 de agosto, 2015

Enviado a corrección: 24 de mayo, 2016

Aprobado: 22 de agosto, 2016 


\section{Introducción}

Existe un aumento de investigaciones en la literatura internacional relacionadas con la modelación matemática ${ }^{3}$ en Educación Matemática. Estas evidencian el nivel de consolidación que tiene la modelación como un dominio de estudio al interior de la Educación Matemática (Blum, Galbraith, Henn y Niss, 2007).

Estas investigaciones reportan, entre otros asuntos, que algunas maneras de hacer modelación en los diferentes niveles escolares posibilitan formas de producir matemática en el aula de clase, estas más articuladas con la cotidianidad de los estudiantes y a través de la relación de sus contextos con los contextos matemáticos escolares. (Araújo, 2002; Barbosa, 2001a; Biembengut y Hein, 2004) Asimismo, indican que la modelación en Educación Primaria permite el desarrollo de las habilidades matemáticas de los estudiantes, el razonamiento matemático y la interpretación de los problemas de la realidad. (Bahmaei, 2013; Biembengut, 2007; English, 2003, 2006, 2009, 2010; Ferreira y Wodewotzki, 2007) Por su parte, Luna y Alves (2007) destacan que a través de la modelación, los estudiantes de educación primaria interactúan con conocimientos matemáticos, con sus usos, y reflexionan de forma crítica a partir de diferentes contextos.

Investigaciones en modelación permiten observar que no hay homogeneidad frente a la comprensión del tema en sus fundamentos epistemológicos y frente a sus posibilidades en el aula de clase (Kaiser y Sriraman, 2006). Estas autoras muestran que las distintas perspectivas dan lugar a posturas variadas; además, presentan una categorización de seis (6) perspectivas: la realista o aplicada, la contextual, la educativa, la socio-crítica, la cognitiva y la epistemológica y teórica. Estas dejan ver intereses investigativos distintos en la modelación; por ejemplo, la realística centra su atención en la solución de problemas del mundo real para entenderlo; la contextual, en resolver problemas del mundo basándose en los contextos y las relaciones; la educativa, en la estructura del aprendizaje y los procesos para su promoción, y en la introducción y el desarrollo de los conceptos; la epistemológica, en promocionar y desarrollar teoría frente a la modelación; y la cognitiva, en el análisis de procesos cognitivos resultantes de la modelación. En contraste, la modelación socio-crítica se centra en la preocupación por el rol de las matemáticas en la sociedad.

La perspectiva socio-crítica reportada por Kaiser y Sriraman (2006) destaca que las potencialidades de la modelación están no solo en enseñar y aprender matemáticas, sino

\footnotetext{
${ }^{3}$ Emplearemos a lo largo de este artículo la palabra modelación para hacer alusión a la modelación matemática en Educación Matemática.
} 
también en la formación ciudadana de los estudiantes. En esa perspectiva, Barbosa (2006) sugiere el uso del término socio-crítico para referirse a una forma de ver la modelación en Educación Matemática, como un reconocimiento a las prácticas pedagógicas que comprenden los ambientes de aprendizaje como una oportunidad para que los estudiantes discutan la naturaleza y papel de los modelos matemáticos en la sociedad.

Silva y Kato (2012) sugieren que la modelación en la perspectiva socio-crítica puede proporcionar oportunidades para que los estudiantes lleven los debates del aula de clase a su vida cotidiana, para crear conciencia con relación con su papel en la sociedad y lograr cambios en la manera que tienen ellos de ver el mundo.

Por su parte, Araújo (2009) se refiere a la modelación en la perspectiva socio-crítica como un espacio donde se propone a los estudiantes, reunidos en grupos, utilizar las matemáticas para resolver algún problema con origen en la realidad; de tal manera que esa resolución sea problematizada y cuestionada. En este sentido, a través de la modelación, se promueve en los estudiantes reflexiones constantes relacionadas con las matemáticas que se utilizan, cómo ellas ayudan o pueden resolver la situación y cómo están inmersas en un sin número de problemáticas con las que se enfrentan a diario.

Autores como Barbosa (2001a, 2001b, 2003a, 2003b, 2006, 2009), Araújo (2002, 2009, 2012), Silva y Kato (2012) destacan algunos elementos que caracterizan la modelación en la perspectiva socio-crítica en los diferentes niveles escolares; ellos han reconocido la participación como una de esas características.

La participación aparece en sus investigaciones como un aspecto fundamental, pues para actuar de forma matemática y crítica frente a las diversas demandas y acontecimientos sociales, se requiere del concurso, compromiso y actuaciones de los diferentes actores involucrados, es decir, se requiere de su participación.

El aula de clase, como una unidad del sistema social, ha de propender porque los estudiantes vivan experiencias en las que participen de situaciones en las cuales se estudien y analicen fenómenos propios de la sociedad y de su cultura; en ese sentido, en el aula de clase de matemáticas la modelación fundamentada en la perspectiva socio-crítica ha de cobrar sentido, pues cuando los estudiantes participan en ambientes de modelación en los que se plantean situaciones relacionadas con problemáticas sociales, reflexionan de las matemáticas involucradas, de cómo las formas de construir modelos matemáticos intervienen en reflexiones sociales y en otros aspectos y conceptos (Barbosa, 2009). Por lo tanto, las matemáticas y los modelos matemáticos construidos pueden servir para analizar y 
tomar decisiones de las situaciones, problemas y fenómenos presentados en el contexto social.

Por su parte, Ferreira y Wodewotzki (2007) plantean algunas reflexiones al respecto de la participación de los estudiantes de educación primaria en situaciones de modelación. Las autoras reconocen que cuando ellos participan en hechos de modelación, se involucran con situaciones próximas a su vida, lo que permite generar más entusiasmo por las matemáticas a partir de la reflexión en torno a las problemáticas sociales.

En particular en ambientes de modelación en la perspectiva socio-crítica y en la educación primaria, se destaca la importancia de la participación de los estudiantes en la construcción del modelo y en la sociedad, mas no se detalla y no se evidencia una preocupación por ella como objeto de investigación.

De las posturas planteadas en los párrafos precedentes, observamos como problemático que, a pesar de llamarse la atención de la importancia de la participación en modelación en la perspectiva socio-crítica y en la educación primaria, hay una exigua cantidad de investigaciones en la educación primaria orientadas a conocer cómo se da la participación de los estudiantes en este marco. En este sentido, desarrollamos una investigación para estudiar y discutir la manera en que un conjunto de estudiantes de quinto grado participó en ambientes de modelación que fueron diseñados con algunas de las características de la perspectiva socio-crítica de la modelación. En particular, en este artículo caracterizamos las interacciones y las contribuciones como una forma de participación de los estudiantes en ambientes de modelación.

Para realizar tal análisis iniciamos el artículo con una discusión teórica de la modelación como ambiente de aprendizaje y de la participación de manera general y en la perspectiva socio-crítica de la modelación. Enseguida, presentamos los aspectos metodológicos de la investigación, y damos un papel especial al contexto donde fue realizada, el enfoque metodológico y los procedimientos para la producción conjunta de registros y datos y para realizar el análisis. Por último, caracterizamos la participación de los estudiantes al involucrarse en ambientes de modelación. 


\section{Referente teórico. La participación en ambientes de modelación matemática en la perspectiva socio-crítica}

De acuerdo con Barbosa (2001a), la modelación se considera un ambiente de aprendizaje que promueve la investigación en otras áreas del conocimiento a través del desarrollo de modelos matemáticos. Así, en ese ambiente, se "invita a los estudiantes a indagar $\mathrm{y} / \mathrm{o}$ investigar, por medio de las matemáticas, situaciones de referencia en la realidad" (Barbosa, 2001a, p. 31)4. En este sentido, la modelación permite a los estudiantes incluir diferentes modos de explicar y comprender la realidad presente en el problema, para luego, abordar y relacionar otras situaciones que se le presenten en su cotidianidad (Barbosa, 2009).

De esta forma, los ambientes de modelación en la perspectiva socio-crítica tienen presente el interés de formar sujetos capaces de actuar de manera activa en la sociedad y de analizar la forma en que las matemáticas son usadas en los debates sociales (Araújo, 2009).

Silva y Kato (2012), por su parte, caracterizaron los ambientes de modelación en la perspectiva socio-crítica en relación con una serie de acciones que los constituyen, que, si bien se vinculan con otras maneras de hacer modelación, cobran en esta postura matices diversos, a saber: i. Participación activa de los estudiantes en la construcción del modelo; ii. Participación activa del estudiante en la sociedad; iii. Problema no matemático de la realidad; iv. Actuación del profesor como mediador.

En los trabajos de Araújo (2002, 2009, 2012), colegimos cinco (5) características de la modelación en la perspectiva socio-crítica: i. Abordar o resolver un problema no matemático de la realidad por medio de las matemáticas; ii. La situación o problema de la realidad es escogido por los estudiantes; iii. Promoción del trabajo en grupo para generar asuntos políticos y democráticos en el aula de clase; iv. Inserción crítica de los estudiantes en su realidad; v. Problematizar el papel de las matemáticas en la construcción del progreso y cuestionar el uso que se ha hecho de ella como instrumento de poder.

Apoyados en la comprensión presentada por Barbosa (2001a, 2001b, 2003a, 2003b, 2006, 2009) de la modelación en la perspectiva socio-crítica, reconocemos, al menos, cuatro (4) características importantes de los ambientes de modelación en esta perspectiva, a saber: i. Situaciones tomadas del cotidiano de los estudiantes que pueden abarcar asuntos de las matemáticas o de otras áreas del conocimiento; ii. Producción de modelos para

${ }^{4}$ Original en Portugués, traducción propia. 
problematizar e indagar en relación con una situación, pero, más allá de eso, que cuestionen las situaciones reales por medio de los métodos matemáticos; iii. Promover espacios de participación de los estudiantes en el trabajo con la situación y en la sociedad; iv. Posibilidad de que ocurran discusiones a través de la interacción y el diálogo entre los estudiantes, y los estudiantes con el profesor.

Las características que hemos expuesto hasta este momento en este artículo presentan elementos comunes que tienen que ver con la situación trabajada, con el análisis del modelo generado, con la participación de los estudiantes y del profesor, y con el cuestionamiento de las ideas matemáticas abordadas en la situación.

En las investigaciones antes mencionadas, la participación aparece como un aspecto fundamental, pues cuando los estudiantes hacen parte de ambientes de modelación en los que se plantean situaciones relacionadas con problemáticas sociales, reflexionan acerca de las matemáticas involucradas, y cómo las formas de construir modelos matemáticos intervienen en reflexiones sociales y de otros aspectos y conceptos (Barbosa, 2009).

En el ámbito educativo, el estudio de la participación ha estado como centro de diversidad de estudios y ha tenido distintas comprensiones; por ejemplo, en un sentido social, la participación se reconoce como la actuación conjunta de un grupo que comparte los mismos objetivos e intereses, es un proceso que reúne al mismo tiempo aprendizaje y enseñanza, pues todos los participantes tienen algo que aportar y algo que recibir; en tal sentido, es a la vez una acción concientizadora y socializante, pues produce una movilización de la conciencia al respecto de las circunstancias de vida, de sus causas y de sus efectos, al mismo tiempo que transmite patrones de comportamiento y nuevas formas de aprender esas circunstancias (Gordillo, 2006).

Wenger (1998) define la participación como el desenvolvimiento del individuo en una práctica, en torno a procesos activos de un grupo de personas que se reúnen para realizar algún tipo de tarea (de la vida cotidiana, el trabajo o la escuela), en acoplamiento mutuo. Entre los miembros del grupo hay un esfuerzo común para llevar a cabo una práctica en la que desarrollan un repertorio compartido de recursos, lenguaje, estilos y rutinas, a través del cual expresan su participación.

En los ambientes educativos con el apoyo de tecnologías, Malinen (2015) ha informado que la forma más común de conceptualizar la participación es la visibilidad en el desarrollo de una actividad. Visibilidad que, por lo general, ha sido operacionalizada en términos de cantidad. La autora reporta que a pesar de que la literatura se ha centrado en la cantidad de 
la participación, en la práctica, la participación puede entenderse como ser un miembro y haber iniciado sesión en un sistema, incluidos los usuarios pasivos, quienes con la sola presencia en el servicio muestran una forma de participación. Sin embargo participar alude al pertenecer. Esta pertenencia trasciende la dicotomía activo-pasivo que se basa en la visibilidad de la actividad, pues se entiende en términos de la calidad de la participación, de la influencia y de hacer contribuciones a la comunidad (Malinen, 2015).

En el aula de clase de matemáticas, de manera particular en el enfoque sociocultural, la participación se centra en el estudiante y en las características del contexto de aprendizaje. La participación ha sido vista como una especie de puesta en común en las prácticas matemáticas que se focaliza en el uso del discurso y de algunos de sus contenidos (normas, valores, valorizaciones) como herramientas mediadoras cruciales con el fin de interpretar y aprender las matemáticas en su contexto (Civil y Planas, 2004).

En este artículo, el escenario elegido son los ambientes de modelación en la perspectiva socio-crítica centrándonos en la participación de los estudiantes de educación primaria. En el siguiente apartado, presentamos los aspectos metodológicos de este escenario.

\section{Metodología. El contexto de la investigación y el camino metodológico}

La investigación se desarrolló en una institución educativa de la ciudad de Medellín, Colombia, en donde se constituyó un semillero de matemáticas, nombrado por los estudiantes protagonistas Cosechando la futura matemática. El semillero funcionó unido a un proyecto institucional denominado Aulas para la Convivencia y la Paz: en mi vida convivo sanamente. En este lugar participan de forma permanente 27 estudiantes de quinto grado (911 años), el profesor de matemáticas del grado, los investigadores y una auxiliar de investigación. Para esto, los estudiantes asistieron durante cuatro (4) meses de su calendario académico (de agosto a noviembre) a 20 sesiones de dos (2) horas cada una.

Nos preocupamos por analizar cómo se dio la participación de los estudiantes de quinto grado cuando se involucraron en ambientes de modelación que siguieron algunas de las características de la perspectiva socio-crítica de la modelación. Para lograr este objetivo, reconocimos, describimos y analizamos las manifestaciones orales y escritas, acciones y explicaciones para luego caracterizar la participación; por tanto, fue importante comprender los asuntos relacionados con el comportamiento de los sujetos en su entorno natural, lo cual dio sentido al uso de un enfoque cualitativo. 
Asumimos el paradigma de investigación cualitativa como un conjunto de prácticas que permiten comprender el comportamiento de los sujetos en relación con el contexto natural al que pertenecen (Bogdan y Biklen, 2007). Aquí, los fenómenos se estudian en el lugar donde se producen, el investigador trata de entenderlos y luego asume una posición frente a ellos.

Empleamos la observación participante, los documentos y las entre-vistas para la producción conjunta de registros y datos. La observación participante es una técnica de investigación de contacto con lo real donde el observador se torna parte de la situación observada e interactúa por grandes periodos de tiempo con los sujetos (Alves-Mazzotti y Gewandsznajder, 1999). Los documentos son cualquier registro escrito que pueda ser usado como fuente de información y fueron producidos de forma paralela a las observaciones (Alves-Mazzotti y Gewandsznajder, 1999). Tomamos como documentos los diarios de campo de la investigación y las producciones escritas de los estudiantes. La entre-vista es un proceso de comunicación que tiene como objetivo obtener descripciones del mundo de la persona entre-vistada con respecto a su interpretación del significado de los fenómenos presentes en la investigación y "se desarrolla como una conver-sación donde los involucrados ven juntos (entre-ven) y co-construyen ideas sobre temas seleccionados" (Skovsmose, Scandiuzzi, Valero, y Alrø, 2011, p. 109).

La producción conjunta de registros y datos se llevó a cabo en un periodo de cuatro (4) meses, tiempo durante el cual los estudiantes formaron parte de tres (3) ambientes de modelación llamados Diseño de una Guía del Consumidor, Cálculo del IMC y Mi proyecto de Modelación.

En términos curriculares, los tres (3) ambientes de modelación estuvieron en correspondencia con los planteamientos de Barbosa (2004) en relación con los casos o maneras de implementar la modelación en el aula de clase en la perspectiva socio-crítica. Lo anterior, ya que, según Barbosa (2001a), la organización de la modelación depende de la experiencia del profesor y de los estudiantes con ella, de los intereses de estos y del contexto escolar.

El primer caso consiste en la problematización de un episodio real, en donde la investigación inicial es del profesor; es él quien formula el problema, recoge los datos cualitativos o cuantitativos y simplifica la información. El estudiante encuentra la solución a partir de la investigación. En el segundo caso, el profesor formula el problema inicial y los estudiantes se enfrentan a la simplificación y a la búsqueda de datos del problema para su posterior solución. En el tercer caso, se abordan proyectos desarrollados a partir de temas 
no matemáticos que pueden ser escogidos por el profesor o por el estudiante. La formulación del problema, la simplificación, la recolección de los datos y la solución son tareas de los estudiantes que se comparten con el profesor.

En correspondencia con los planteamientos de Silva y Kato (2012), Araújo (2002, 2012, 2009), Barbosa (2001a, 2001b, 2003a, 2003b, 2004, 2006, 2009) asumimos cinco (5) características de la perspectiva socio-crítica de la modelación para el diseño de los ambientes de modelación.

La primera hace referencia a que las situaciones fueron tomadas de la cotidianidad del estudiantado y abarcaron de manera igual asuntos y conocimientos de las matemáticas y de otras áreas del conocimiento. La segunda hace alusión a la promoción de asuntos políticos y democráticos al interior del aula de clase a partir del trabajo en grupo. La tercera se refiere a cómo los modelos matemáticos construidos permitieron a los estudiantes problematizar, discutir e indagar acerca de la situación abordada; los discentes expusieron, criticaron y reformularon los modelos creados para determinada situación y consideraron la posibilidad de que el modelo construido sirviera para analizar y tomar decisiones de determinada situación. La cuarta característica se refiere a espacios de participación de los estudiantes en el trabajo con la situación y en la sociedad. Ellos se preocuparon por analizar y cuestionar la situación, lo que hizo que se involucraran de manera activa y que el trabajo realizado pasara, en algunos casos, los límites del aula de clase. La quinta alude al papel de los investigadores como mediadores que promovieron en los alumnos el trabajo en conjunto, estimularon la exposición de ideas y argumentos e hicieron del aula de clase un espacio democrático en el que todos tienen condiciones iguales de trabajo.

En cada uno de los ambientes de modelación, analizamos cómo los estudiantes participaron al involucrarse, comprometerse y apropiarse de la situación, al reflexionar de su entorno, sus necesidades y las decisiones que debían tomar en relación algo, y al generar sus ideas y procesos matemáticos.

En el primer ambiente de modelación, los estudiantes diseñaron guías del consumidor ${ }^{5}$ para decidir cuáles aspectos deberían tener en cuenta a la hora de elegir un producto. En el segundo ambiente de modelación, analizaron el modelo del índice de Quetelet o Índice de Masa Corporal $(I M C)^{6}$, que es el indicador más utilizado para evaluar el nivel de obesidad o

\footnotetext{
${ }^{5}$ Algunos cambios se realizaron al original, el cual se encuentra en: English (2006).

${ }^{6}$ Las necesidades que emergieron en el diseño del ambiente de modelación en la perspectiva sociocrítica nos llevaron a convocar a una auxiliar de investigación y a una nutricionista dietista. Juntos diseñamos el ambiente de modelación propuesto.
} 
delgadez de una persona o comunidad. En el tercer ambiente de modelación, formaron grupos de trabajo en los cuales propusieron una situación problema de su interés para ser estudiada por medio de las matemáticas. Cada uno de los ambientes de modelación se desarrolló en cinco (5) momentos en los cuales los estudiantes se vieron enfrentados a modelar matemáticamente. Los momentos fueron: describir e interpretar la situación, simplificar asuntos en la situación, recolectar información pertinente para la situación, proponer una solución o soluciones a la situación y comunicar los resultados.

En este artículo discutiremos y analizaremos la participación en los dos (2) primeros ambientes propuestos. Mostramos en la Tabla 1 la secuencia completa de estos ambientes. En los diferentes momentos de los ambientes de modelación propuestos buscamos la problematización y la investigación. Problematizar es crear preguntas y levantar cuestiones acerca de problemas de la realidad. Investigar es un proceso de búsqueda, selección, organización y manipulación de la información. (Barbosa, 2004)

Tabla 1

Secuencia de ambientes de modelación matemática

\begin{tabular}{lllll}
\hline Momentos & \multicolumn{2}{c}{$\begin{array}{c}\text { Diseño de una guía del } \\
\text { Consumidor }\end{array}$} & \multicolumn{1}{c}{ Cálculo del IMC } \\
\hline $\begin{array}{l}\text { Descripción e interpretación } \\
\text { de la situación por Estudiar }\end{array}$ & $\begin{array}{l}\text { ¿Qué es una guía del } \\
\text { consumidor? }\end{array}$ & $\begin{array}{l}\text { Obesidad y delgadez en el } \\
\text { país }\end{array}$ \\
$\begin{array}{l}\text { Simplificación de asuntos } \\
\text { por considerar en la la } \\
\text { situación }\end{array}$ & $\begin{array}{l}\text { Observación de los paquetes de } \\
\text { mecato. Criterios previos de } \\
\text { clasificación }\end{array}$ & Cálculo del IMC \\
$\begin{array}{l}\text { Buscar información } \\
\text { pertinente para la situación }\end{array}$ & $\begin{array}{l}\text { Buscar información pertinente } \\
\text { para la situación }\end{array}$ & Diálogo con una nutricionista. \\
Clasificación del IMC
\end{tabular}

Fuente: Elaboración propia de los autores (2015).

Según Miles y Huberman (1994, citados por Alves-Mazzotti y Gewandsznajder, 1999), el análisis de los datos se configura dentro de un proceso de reducción, focalización, organización e interpretación de los datos en el fenómeno que se estudia. 
La información que recogimos se estudió y se organizó con el propósito de identificar cómo se dio la participación de los estudiantes en los dos (2) ambientes de modelación seleccionados. Asumimos como unidades de análisis las manifestaciones orales y escritas, las acciones y las explicaciones de los estudiantes cuando se involucraron en los ambientes de modelación.

\section{Resultados y su análisis: formas de participación de los estudiantes de quinto grado en ambientes de modelación matemática}

En este apartado presentamos los resultados del análisis de la información acerca de las características de la participación de los estudiantes cuando modelan matemáticamente; es decir, cuando describen e interpretan las situaciones o fenómenos por estudiar, matematizan las relaciones propias de la situación, interpretan y analizan los resultados.

\subsection{La descripción e interpretación del ambiente de modelación matemática como generadora de interacciones}

Cada uno de los ambientes de modelación inició con una descripción e interpretación de la situación que se deseaba estudiar, esta descripción es un proceso que consistió en que los estudiantes reconocieran las características propias de las situaciones o fenómenos por estudiar para develar el problema por estudiar.

La descripción e interpretación de la situación se nombró en el primer ambiente "¿Qué es una guía del consumidor?" y en el segundo ambiente "Obesidad y delgadez en el país".

A continuación, describiremos cómo se dio este proceso en los dos (2) ambientes de modelación.

\subsection{1 ¿Qué es una guía del consumidor?}

El primer ambiente de modelación inició con la lectura del documento de las guías del consumidor en Colombia, expedido por la Superintendencia de Industria y Comercio (SIC) ${ }^{7}$. Una vez terminada la lectura del documento, realizamos un diálogo con los estudiantes frente a ¿qué es un consumidor?, ¿qué se considera un artículo de consumo?, ¿qué criterios puede considerar una persona a la hora de adquirir un producto?, ¿qué es una guía del consumidor? En la Tabla 2 mostramos respuestas dadas por algunos estudiantes:

${ }^{7}$ El documento se recuperó el 10 de junio de 2014 de la página de la Superintendencia de Industria y Comercio (SIC): http://www.sic.gov.co/recursos_user/documentos/atencion_usuario/Guia_Consumidor.pdf 
Tabla 2

Diálogos de la descripción e interpretación de la situación por estudiar

\begin{tabular}{|c|c|c|c|c|}
\hline $\begin{array}{l}\text { ¿Qué es } \\
\text { consumidor? }\end{array}$ & $\begin{array}{l}\text { ¿Qué se considera } \\
\text { un artículo de } \\
\text { consumo? ¿Quiénes } \\
\text { consumen? }\end{array}$ & $\begin{array}{l}\text { ¿Qué criterios puede } \\
\text { considerar una } \\
\text { persona a la hora de } \\
\text { adquirir un } \\
\text { producto? }\end{array}$ & $\begin{array}{l}\text { ¿Qué es una guía del } \\
\text { consumidor? }\end{array}$ & \\
\hline $\begin{array}{l}\text { Es el que gasta, como } \\
\text { agua, y le dicen cuánta } \\
\text { agua consumió. Es } \\
\text { alguien que adquiere algo, } \\
\text { que disfruta de algo y eso } \\
\text { se va acabando. }\end{array}$ & $\begin{array}{l}\text { Profe todas las cosas } \\
\text { de la tierra, porque } \\
\text { todo se va acabando. }\end{array}$ & $\begin{array}{l}\text { Profe, mirar bien las } \\
\text { cosas del producto. } \\
\text { Profe sí, además si } \\
\text { los alimentos de los } \\
\text { que está hecho nos } \\
\text { hacen daño. }\end{array}$ & $\begin{array}{l}\text { Entonces ahí van a } \\
\text { escribir una lista de } \\
\text { todas las cosas que } \\
\text { debe de tener en } \\
\text { cuenta. } \\
\text { Cosas que debe tener } \\
\text { en cuenta un } \\
\text { consumidor. }\end{array}$ & Juan José \\
\hline \multirow[t]{2}{*}{$\begin{array}{l}\text { El que compra y gasta el } \\
\text { dinero. }\end{array}$} & & $\begin{array}{l}\text { Las calorías, los } \\
\text { precios. }\end{array}$ & & Tomás \\
\hline & 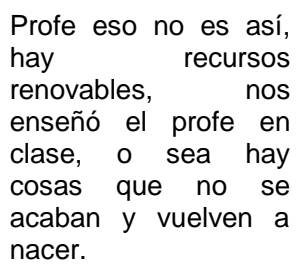 & $\begin{array}{l}\text { Profe, pues según lo } \\
\text { que leímos que nos } \\
\text { respeten los } \\
\text { derechos, a que no } \\
\text { nos obliguen qué } \\
\text { comprar. De qué está } \\
\text { hecho, si está bueno, } \\
\text { si cumplió las normas. }\end{array}$ & & Paulina \\
\hline $\begin{array}{l}\text { Profe es la persona que } \\
\text { adquiere algún beneficio. }\end{array}$ & $\begin{array}{l}\text { El Sol consume } \\
\text { energía, las plantas } \\
\text { consumen al sol, los } \\
\text { gatos consumen } \\
\text { agua, las personas } \\
\text { consumimos cosas } \\
\text { como la ropa, profe } \\
\text { todos consumimos. }\end{array}$ & $\begin{array}{l}\text { La plata, el valor de } \\
\text { producto. }\end{array}$ & $\begin{array}{l}\text { Una orientación para } \\
\text { comprar. }\end{array}$ & Valentina \\
\hline
\end{tabular}

En las intervenciones algunos estudiantes respondieron a la pregunta de los investigadores y plantearon de manera abierta sus conocimientos, al describir y explicar los diferentes asuntos que intervienen en la situación que modelaban. Mientras otros optaron por el silencio, pero evidenciaron atención al trabajo realizado.

En este primer momento, se evidenció un compromiso parcial de los estudiantes con el tema, que se relaciona con una reacción natural de ellos a las preguntas presentadas por los investigadores. Aquí la participación está caracterizada por una marcada influencia de los profesores quienes plantean las preguntas y los estudiantes pueden reaccionar de diferentes maneras, el acto participativo es propuesto por los investigadores que impulsan a los estudiantes a participar. 
Para organizar el trabajo y ampliar el conocimiento de los estudiantes frente a la situación, delimitamos los consumidores a consumidores de mecato ${ }^{8}$. En este momento se organizaron en grupos de trabajo (3 o 4 estudiantes) y cada estudiante recibió un (1) paquete distinto de mecato para que decidiera qué aspectos observar en él y luego escribiera los elementos que identificara con relación al producto. Trascripciones de algunas de las producciones escritas se muestran en la Tabla 3.

Tabla 3

Observaciones a los paquetes de mecato

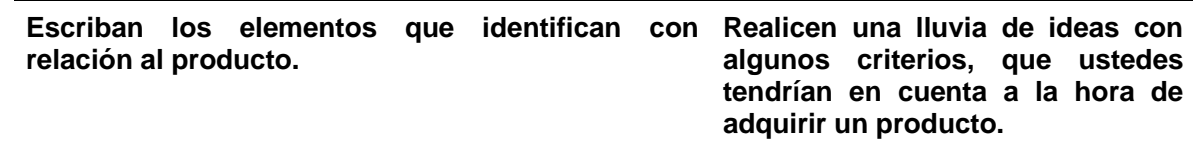

Observamos el peso, el lote y la fecha de
vencimiento. Porque hace poco una compañera se comió algo que estaba pasado [vencido] y la tuvieron que llevar al médico.

Nosotros pensamos que lo importante es tener en Carga nutricional, fecha de Grupo 3 cuenta el tipo de alimentos que tiene el producto, y vencimiento, tabla de alimentos 0

saber cuánta grasa aporta, para así saber si nos hace ingredientes, modo de fabricación. daño o no.

Fecha de vencimiento, peso, sabor. Grupo 2

Atención al cliente, fecha de Grupo 4

A nosotros nos gusta mucho el medioambiente, por eso observamos que el paquete tiene harina de trigo fortificada, aceite vegetal y una invitación a cuidar el medio ambiente. vencimiento, contenido del producto.

No sabíamos cómo escoger las categorías, pero Paulina sabe mucha matemática y ella nos explicó.

Precio por unidad, fecha de Grupo 8 vencimiento, país de fabricación.

Entonces nos fijamos en el precio al que lo venden.

Fuente: Elaboración propia de los autores (2015).

En este momento, los estudiantes participaron al dar sugerencias de qué observar, qué considerar y qué analizar frente al diseño de una guía del consumidor. Tomaron decisiones frente a qué observar y argumentaron las decisiones, para lo que tuvieron en cuenta lo que han escuchado en la escuela o fuera de ella o lo que ellos y sus padres hacen en casa. También, según lo que observan, no saben y les genera curiosidad.

Aquí la participación se caracteriza por un compromiso más amplio, los estudiantes empezaron a configurar sus criterios de elección (Gordillo, 2006) cuando comenzaron a tomar parte en las decisiones de compra o las realizaron de forma directa.

\footnotetext{
8 Los estudiantes de la institución comprenden que mecato es un dulce, golosina o comida ligera de diferentes sabores.
} 
Las observaciones hechas por los estudiantes fueron dadas a conocer en el semillero y, a partir de ellas, cada grupo de trabajo estableció criterios que podrían considerarse a la hora de diseñar la guía para la compra de los mecatos. En la puesta en común surgieron diferentes criterios por considerar en el diseño de la guía, así que algunos grupos de trabajo propusieron unificar los criterios, situación que fue avalada por el común del grupo.

Así, cuestionamos a los estudiantes según qué criterios incluir a la hora de diseñar la guía y por qué. En la Tabla 4 presentamos algunas de las manifestaciones del grupo.

Tabla 4

Criterios previos de clasificación

\begin{tabular}{|c|c|c|}
\hline $\begin{array}{c}\text { ¿Qué criterios incluirías en el diseño de la guía del } \\
\text { consumidor? }\end{array}$ & ¿Por qué eliges ese criterio? & \\
\hline Fecha de vencimiento & $\begin{array}{l}\text { Porque yo me comí algo que ya } \\
\text { estaba vencido, entonces sería } \\
\text { bueno que las personas se fijen en } \\
\text { eso. }\end{array}$ & Sofía \\
\hline Tabla de alimentos & $\begin{array}{l}\text { Debemos saber qué tipo de } \\
\text { alimentos contiene para saber si es } \\
\text { saludable para nosotros o no, y así } \\
\text { saber cuánto podemos comer. }\end{array}$ & Valentina \\
\hline Carga nutricional & $\begin{array}{l}\text { Porque esto nos indica el consumo } \\
\text { calórico y la cantidad de energía que } \\
\text { esto nos aporta en nuestra vida. }\end{array}$ & Santiago \\
\hline Atención al cliente & $\begin{array}{l}\text { Porque como compradores tenemos } \\
\text { derecho a que nos escuchen } \\
\text { nuestras quejas e inquietudes. }\end{array}$ & Paulina \\
\hline Precio por unidad & $\begin{array}{l}\text { Para poder saber qué es más } \\
\text { económico para nosotros. }\end{array}$ & Juan José \\
\hline
\end{tabular}

Fuente: Elaboración propia de los autores (2015).

Se eligieron 10 criterios previos de clasificación comunes a todo el semillero: fecha de vencimiento, carga nutricional, peso neto, atención al cliente, tabla de alimentos o ingredientes, contenido del producto, precio por unidad, sabor, modo de fabricación y país de fabricación.

Los estudiantes argumentaron sus posturas acerca de los criterios por incluir. Lo anterior demostró una motivación para actuar. Las contribuciones y el ser propositivos frente a una situación dio cuenta en los estudiantes de una participación más activa (Gorgorió y Prat, 2009).

Al compartir sus ideas, se animó a los estudiantes a negociar, discutir, escuchar a los demás y respetar sus opiniones. "Esta es una forma de trabajar los temas políticos y de la democracia en la micro sociedad del aula" (Araújo, 2009, p. 65) ${ }^{9}$.

\footnotetext{
${ }^{9}$ Original en Portugués, traducción propia.
} 
Esta participación fomenta en los estudiantes la expansión de la autonomía, que tiene como objetivo proporcionar la lectura y expansión de la visión del mundo, el desarrollo del pensamiento autónomo y contribuir al pleno ejercicio de la ciudadanía (Araújo, 2009).

\subsubsection{Obesidad y delgadez en el país}

En el segundo ambiente de modelación pretendimos concientizar a los estudiantes de los problemas de obesidad y delgadez en Colombia y su prevención, así como discutir alcances y usos de los modelos matemáticos.

El ambiente inició con discusiones en torno a las estadísticas reportadas de la cantidad de personas en Colombia con obesidad. Al respecto, algunos estudiantes manifestaron: "profes, lo que pasa en el país es grave, las encuestas dicen que uno (1) de cada dos (2) colombianos es obeso, esto es un valor muy alto para el país, porque es la mitad", "profe, el porcentaje nos indica lo que valen esos departamentos relacionados con Colombia", "profe, tenemos un gran problema por la obesidad, entonces las personas se van a enfermar más". "profes, debería hacerse algo sobre esto, que todos estemos más comprometidos a alimentarnos bien para tener una buena salud" (Verbalizaciones de los estudiantes, 14 de octubre del 2014).

Lo anterior permitió reconocer los riesgos para la salud y la problemática por la que pasa el país a nivel nutricional, así como identificar algunos asuntos matemáticos como el porcentaje y la estadística. A partir de las estadísticas presentadas, reconocimos el IMC como un índice que proporciona una medida del sobrepeso y la delgadez y que sirve como factor para determinar los rangos presentados.

Una vez discutidos los primeros asuntos, los estudiantes realizaron el cálculo del IMC propio. Para el cálculo del IMC trabajaron en grupos y emplearon el modelo matemático de Quetelet, modelo igual para todas las personas:

$$
I M C=\frac{\text { Peso }(\mathrm{Kg})}{\text { Estatura }^{2}(\mathrm{~m})}
$$

Aquí reflexionamos acerca de lo que es el IMC, para qué y cómo puede ayudar en el diario vivir de las personas. Así, discutimos, por ejemplo, que el IMC es el índice de masa corporal, kilogramos por metro cuadrado, que es el índice que mide la dimensión corporal, el cual indica el área ocupada por el cuerpo en el espacio.

En este cálculo, los estudiantes se confundieron al tomar su estatura al cuadrado y el peso en kilogramos, y expresaron asuntos como: "vamos a medir nuestro IMC en kilogramos 
o cuadrados" (Verbalizaciones de los estudiantes, 16 de octubre del 2014). Sin embargo, hubo otros que comprendieron la medición en centímetros o metros y en kilogramos. Lo anterior permitió que se diera un diálogo para tomar decisiones y se llegara a acuerdos en cuanto a las unidades de medida del IMC. Una vez obtenido el valor numérico para el IMC, los estudiantes se inquietaron por conocer cuál significado tienen los valores.

Para interpretar el número obtenido al calcular el IMC les presentamos los valores de clasificación de la Organización Mundial de la Salud (OMS), que describen el IMC en la población de hombres y mujeres mayor de 18 años; por ser las interpretaciones más encontradas en los medios. En este punto, uno de ellos manifestó: "pero todos nosotros estamos muy, muy abajo del IMC adecuado, ¿por qué pasa esto?" (Verbalizaciones de los estudiantes, 21 de octubre del 2014). Los discentes notaron que su IMC es muy bajo en comparación con los valores dados, y que al parecer se encuentran bajos de peso.

En este momento les preguntamos si existen interpretaciones diferentes para el valor obtenido en el cálculo del IMC. Para resolver esta inquietud, fue necesario que indagaran otros criterios generados por la OMS. Al respecto, uno de ellos investigó y contó al grupo: "la OMS tiene tablas diferentes para las edades y los géneros, nosotros debemos usar otras tablas". (Verbalizaciones de los estudiantes, 21 de octubre del 2014)

Con ayuda de la nutricionista dietista, los estudiantes reportaron que la OMS utiliza el IMC/Edad como único parámetro para analizar la relación del peso y la estatura en el grupo de edades de 5 a 18 años. El valor hallado para el IMC es analizado en las gráficas de $I M C / E d a d$, las cuales varían según la edad y el género. Esta variación se da porque existen diferencias relacionadas con el sexo, que son evidentes en el momento de nacer y en la pubertad. De esta manera, fue posible que clasificaran los valores obtenidos por ellos, involucrándose en la indagación de los estándares establecidos por la OMS para determinar sus principales características.

A partir de este trabajo, reflexionaron en torno a problemáticas sociales en donde las matemáticas son fundamentales para dar respuesta a muchos interrogantes, entre ellas el IMC. Lo anterior evidenció una participación caracterizada por la reflexión de los estudiantes, pues les demandó tomar datos auténticos de la situación, procesar dichos datos, aplicar un modelo matemático y dar a conocer los procedimientos y los resultados con sus compañeros de clase. En este sentido, el grupo se involucró de manera directa en la búsqueda de la solución del problema del peso y la alimentación y en las discusiones de este, más allá de 
los límites de la clase, lo que implicó un mayor compromiso de los estudiantes con problemas externos a la escuela (Araújo, 2009).

Los estudiantes encontraron en el cálculo del IMC asuntos matemáticos y nutricionales en torno a los cuales hicieron una reflexión con el fin de aproximarse a una posible solución a las problemáticas presentadas. De esta manera, identificaron que las matemáticas pueden dar indicios de si una persona presenta obesidad o delgadez, sin dejar de lado la idea de que el modelo no es absoluto, pues tiene, como todos los modelos, un campo de aplicación y campos en los cuales no puede ser aplicado. Frente a lo anterior, la perspectiva sociocrítica, según Barbosa (2001a), enfatiza en reconocer hasta qué punto pueden o no usarse los modelos en la sociedad.

\subsection{Proponer una solución o soluciones a la situación. Contribuciones a partir de la matematización}

En los ambientes de modelación que se desarrollaron, los estudiantes iniciaron procesos de matematización. Todo esto dio lugar a una preocupación por las matemáticas como una forma de entender la situación y de ofrecer una solución que sirve en la realidad. A continuación, describiremos cómo se dio el proceso tanto en la situación de la guía del consumidor como en la del IMC.

\subsection{1 ¿Qué es una guía del consumidor?}

En el primer ambiente de modelación, los estudiantes resaltaron que el nombre de los criterios por si solo es insuficiente para construir la guía, que es necesario indagar su significado. Buscaron información adicional de los criterios para tomar decisiones con base en cuáles usar o no. Indagaron en grupos de trabajo los 10 criterios previos a partir de entrevistas a sus profesores y familiares y de consultas en internet.

Al hacer las investigaciones, los grupos de trabajo identificaron, entre otros elementos, que en el país existen leyes que reglamentan los criterios previos que elegimos y se interesaron en algunos. A continuación, ejemplificamos las indagaciones efectuadas por los estudiantes y la problematización que se generó.

Un grupo descubrió que algunos de los criterios que presentamos en el aula de clase son tenidos en cuenta por la SIC para generar guías para consumidores; por ejemplo, frente al criterio precio por unidad encontraron que "en la actualidad, todos los bienes susceptibles 
de ser vendidos por unidad de medida, es decir, por cantidad de masa, volumen o longitud deben indicar el precio por unidad de medida" (SIC, p. 26).

En esta indagación reflexionamos con los estudiantes que el precio por unidad de medida es útil cuando, por ejemplo, un mismo producto es vendido en presentaciones de diferentes tamaños, pues permite identificar el más económico, que no implica que sea el que aparece con un precio de venta inferior. Los estudiantes discutieron esta afirmación y presentaron sus argumentos al respecto.

En la Figura 1 mostramos la comparación realizada por dos (2) grupos de trabajo de un producto que es ofrecido en el país en tres (3) presentaciones diferentes.

\section{Figura 1}

Comparación de productos. Producción escrita de los grupos 1 y 3.
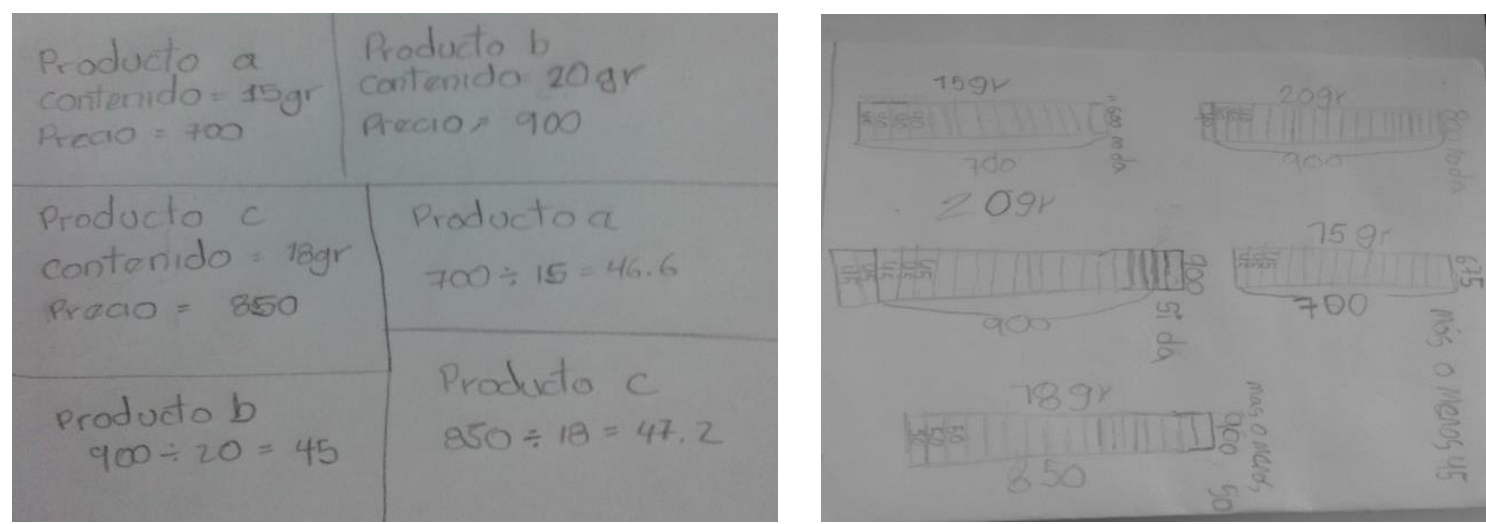

Fuente: Producciones de los estudiantes (2014).

Ambos grupos de trabajo reconocieron que para saber la relación entre el precio y el contenido es necesario comparar el costo del producto y la cantidad ofrecida en el producto, para determinar cuánto vale la unidad de esa cantidad ofrecida. En la comparación, emplearon las matemáticas como una herramienta para dar respuesta a su problema.

Otro de los grupos discutió acerca de la carga nutricional que aparece en los productos. Indagaron que allí se explicita la información del contenido y las propiedades nutricionales del alimento.

En este punto, les cuestionamos acerca de las características generales que deben ir en el rotulador nutricional. Juntos construimos un esquema, mostrada en la Figura 2, que contiene las características nutricionales comunes en todos los paquetes. Al estudiar la significación de cada uno de los componentes del esquema, cuestionamos las características de cada uno de ellos. 
Figura 2

Valores de referencia de nutrientes (VRN)

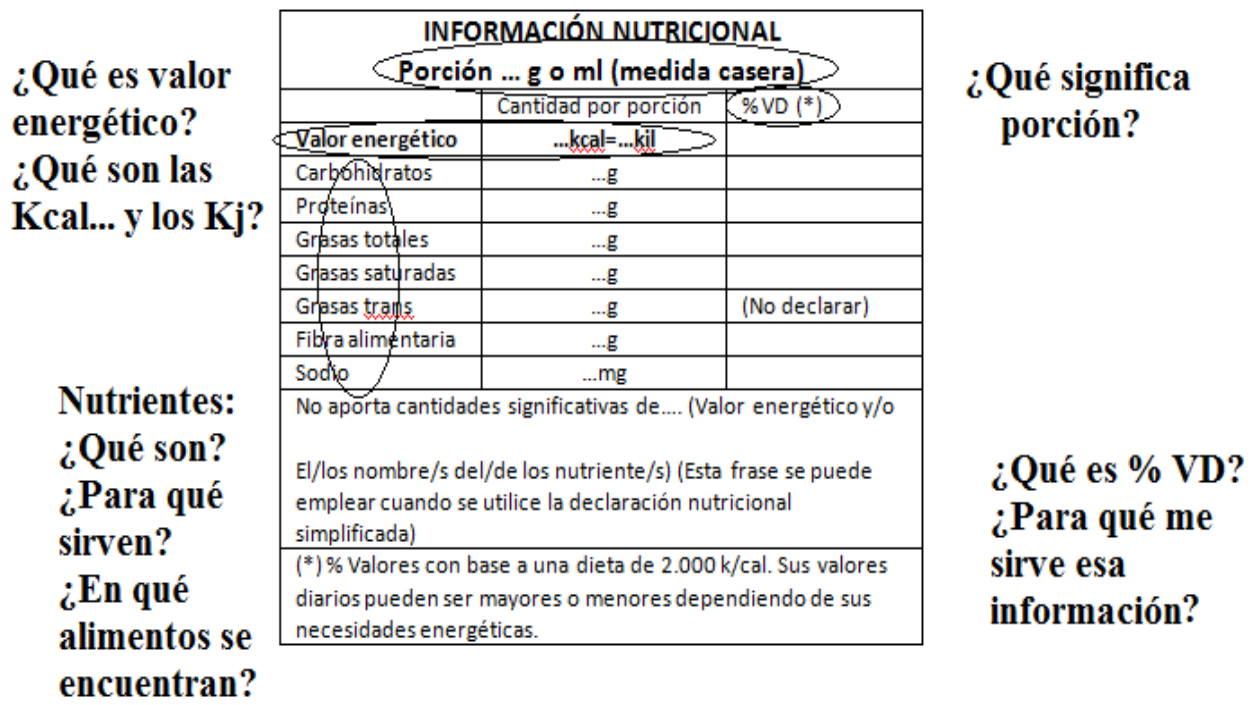

Fuente: Producciones de los estudiantes (2014).

Uno de los grupos manifestó interés por los Valores de Referencia de Nutrientes (VRM), así que preguntamos a los estudiantes por el significado de los valores, en especial, por el porcentaje que cubre una porción del alimento que contiene cinco (5) gramos de fibra. Un grupo realizó una representación simbólica para analizar la situación propuesta. En los estudiantes que aquí mencionamos, la idea de incógnita aparece como una manera de modelar una relación de proporcionalidad entre dos (2) cantidades. Mostramos dicha representación en la Tabla 5.

Tabla 5

VRN para un producto. Trascripción del trabajo realizado por el grupo 2

\begin{tabular}{|cc|}
\hline & $\begin{array}{c}25 \text { gramos de fibra }=100 \% \text { del VRN } \\
5 \text { gramos de fibra }=x\end{array}$ \\
$x=\frac{100 * 5}{25}=20$ & \\
\hline
\end{tabular}

La búsqueda de información y el trabajo en equipo fueron importantes en la preparación del modelo que requirió una actitud participativa en la cual se mostraron curiosidad y creatividad. 
Durante la matematización, les dimos voz, lo que les hace sentir que su participación es importante para el desarrollo de la actividad y para la comprensión entre sus compañeros.

La participación aparece al formar parte de la situación, cuando los estudiantes se implicaron en el análisis de la información que se les ofreció (Gordillo, 2006). En este caso, participaron con el compromiso como consumidores, al procurar conocer todas las alternativas existentes de productos, clasificar de forma correcta cada una en términos de ventajas y desventajas, e identificar las mejores alternativas.

Basados en la discusión de las investigaciones de los 10 criterios previos, cada grupo de trabajo eligió cuatro (4) criterios de clasificación y elaboró la guía que debería tener en cuenta un consumidor a la hora de hacer una compra. Para esta elección, tuvieron en cuenta las matemáticas como una herramienta para dar explicación al porqué incluir cada criterio.

Los estudiantes asumieron la creación de las guías como un problema que cualquier persona podría enfrentar en el día a día. Esta experiencia favoreció su motivación y su interés para ofrecer una posibilidad de orientar a un consumidor. Lo anterior se consolidó como una forma de participación. Se puede ver en el trabajo de los estudiantes que la modelación propició la construcción de diferentes representaciones que están vinculadas a asuntos matemáticos y a su contexto cercano, lo que indica que la modelación puede ayudar en la exploración y construcción de significado a los conceptos matemáticos.

\subsubsection{Obesidad y delgadez en el país}

En el segundo ambiente de modelación, se contó con la presencia de una profesional en Nutrición y Dietética con quien problematizamos los valores numéricos obtenidos con el cálculo del IMC y clasificamos los resultados numéricos del $I M C$ en las gráficas IMC/Edad para hombres y mujeres entre los 5 y los 18 años, establecidas por la OMS.

Para clasificar esta información, en primer lugar, explicamos el cálculo de la edad, según el número de años y meses cumplidos; este fue de fácil comprensión. A continuación, mostramos en la Tabla 6 el cálculo realizado por Tomás. 
Tabla 6

Cálculo de la edad. Trascripción de producción escrita de Tomás el 21 de octubre de 2014

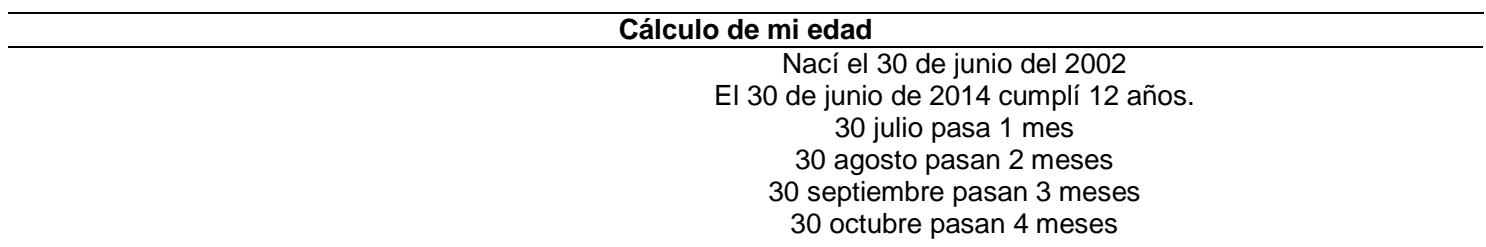

Entonces tengo 12 años y 3 meses porque hoy es 21 de octubre.

Fuente: Elaboración propia de los autores (2015).

Al clasificar la información en las gráficas, los estudiantes realizaron discusiones matemáticas y nutricionales en torno a los resultados del cálculo del IMC.

En segundo lugar, dialogamos con los estudiantes de la lectura de las gráficas; ellos expresaron la similitud entre las gráficas de crecimiento con el plano cartesiano. Con el cálculo de los componentes se trazaron las líneas que salen de cada eje y se procedió a verificar el punto donde se interceptaron. La relación se muestra en la Figura 3.

Figura 3

Ubicación del IMC. Producción de los estudiantes

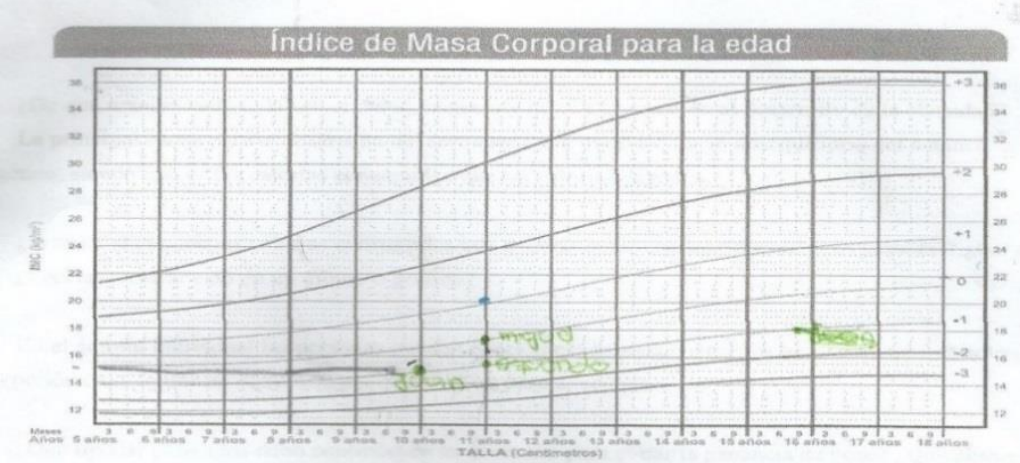

Fuente: Producciones de los estudiantes (2014).

Algunos estudiantes notaron que su peso corporal era diferente al arrojado por los cálculos; y muchos manifestaron que iban a realizar el ejercicio con otros compañeros y con familiares, ya que les pareció algo fácil de hallar y que sería útil que diferentes personas lo conocieran. Algunos realizaron cuestionamientos a sus prácticas alimenticias, lo que los llevó a pensar y actuar acerca de sus hábitos en pro de una mejor salud. Sus interacciones pusieron en evidencia una participación espontánea, que generó discusiones matemáticas grupales. 
Basados en las lecturas e interpretaciones realizadas por los estudiantes frente a la clasificación de su IMC, problematizamos el modelo matemático para el cálculo del IMC, y los cuestionamos por asuntos como: ¿será útil este cálculo para todas las personas?, ¿el cálculo es exacto?, ¿es suficiente este cálculo para determinar si una persona tiene sobrepeso o delgadez?, ¿qué cuestiones hay que tener en cuenta a la hora de dar un diagnóstico a una persona?, ¿qué asuntos matemáticos son cuestionables en el cálculo?

De acuerdo con el análisis del modelo para el cálculo $I M C$, a las discusiones con los investigadores y al cálculo de su propio $I M C$, los estudiantes plantearon diversos asuntos matemáticos y nutricionales en torno al modelo matemático de Quetelet, los cuales detallamos en la Tabla 7.

Tabla 7

Asuntos analizados al modelo del IMC

\begin{tabular}{l} 
Asuntos nutricionales \\
\hline El modelo plantea criterios de delgadez y obesidad sin \\
tener en cuenta la composición corporal; o sea, los \\
componentes del peso corporal.
\end{tabular}
componentes del peso corporal.

El modelo crea un impedimento cuando tenemos que evaluar deportistas de alto rendimiento, que pueden tener un alto valor en el peso corporal; pero a partir de un alto porcentaje de músculos y de masa corporal activa en general y bajos niveles de grasa.

Como nutricionalmente se sabe que el cálculo no es exacto, es necesario recurrir a otros asuntos nutricionales, como la composición corporal, para determinar si el peso obtenido es dado por tejido muscular o por tejido graso.

Fuente: Elaboración propia de los autores (2015).

\begin{abstract}
Asuntos matemáticos
El modelo no es muy preciso y podría presentar errores pues relaciona medidas de dimensiones distintas, ya que, el peso es tridimensional y la altura es de una dimensión y aunque se eleve al cuadrado, no se iguala la dimensión, y por lo tanto los cálculos realizados no son correctos.
\end{abstract}

Los instrumentos de medida que empelamos no son los más adecuados, y generan margen de error alto.

Es necesario desarrollar otro índice de masa corporal que sea más exacto.

El modelo matemático usado apareció como una manera de generar primeros indicios del estado nutricional, pero se definió que requiere de una valoración profunda por los especialistas. Además, la precisión del modelo se relaciona con dos (2) aspectos: el primero en relación con la no precisión de los instrumentos de medida, y el segundo en relación con la falibilidad que tienen por naturaleza los modelos matemáticos.

En los asuntos planteados por el grupo, se hizo evidente que empezaron a reflexionar acerca de nociones matemáticas, preocupándose por las matemáticas como forma de entender asuntos propios de su realidad, lo que promovió en ellos la participación. Es de anotar que a pesar de que los estudiantes encontraron algunas dificultades con el modelo, comprendieron la importancia del análisis más allá del resultado que arroja el cálculo del 
IMC, para tomar posición con respecto a lo que nos compete; en este caso, si se sufre de obesidad o delgadez. De igual forma, experimentaron que a través del análisis de un modelo matemático pueden establecerse parámetros para la salud. Estos se definen a nivel individual al hallar y clasificar el IMC; a nivel grupal al analizar los porcentajes epidemiológicos propuestos por la OMS. Aquí, la modelación apareció como una estrategia para analizar y valorar los modelos, lo que empezó a generar en los estudiantes una postura diferente frente a las matemáticas en situaciones cercanas a ellos.

\section{Conclusiones}

El objetivo de este artículo fue analizar y caracterizar, a partir de las manifestaciones orales y escritas, las acciones y las explicaciones de los estudiantes, las interacciones y las contribuciones como una forma de participación. La participación fue analizada en las formas de actuar y de comprometerse de ellos cuando se enfrentaron a la modelación en la perspectiva sociocrítica de la modelación.

Del análisis de los momentos surgió que cuando los estudiantes se involucran en este tipo de ambientes de modelación, su participación se caracteriza por las interacciones y las contribuciones que se asumen como elementos que ayudan a construir las comprensiones necesarias para el aprendizaje en el discurso matemático del aula de clase.

Las interacciones son las acciones de socializar ideas y compartir con los demás puntos de vista, conocimientos, reflexiones, sentimientos, hallazgos y posturas con respecto a un objeto de estudio. De este modo, las interacciones no son solo un mensaje y una respuesta, sino una serie de discusiones espontáneas y coherentes entre estudiantes, con o sin la participación del profesor, para lo cual es necesario que la actividad propuesta en los ambientes se oriente a fomentar el análisis de diversos puntos de vista y la toma de posición al respecto.

Asimismo, las contribuciones se conciben como los aportes a las discusiones y a la actividad que se realiza, estas van más allá de estoy de acuerdo o no me parece; y se vinculan mucho más con los aportes productivos, que agregan valor a lo que se discute, que ayudan a otros a expresar lo que piensan y a explorar nuevas áreas.

Los resultados de este estudio muestran que los ambientes de modelación en educación primaria diseñados en la perspectiva socio-crítica favorecen espacios de diálogo y acción hacia una participación reflexiva y propositiva de los estudiantes. Para que la participación sea cada vez más reflexiva y propositiva y llegue a incidir en la realidad de los 
estudiantes, se necesita promover y facilitar ambientes que favorezcan la comunicación, el diálogo, y el compromiso entre los participantes.

Además, las diferentes formas de actuar y de comprometerse de los estudiantes en los ambientes de modelación propuestos dejan ver cómo comunican sus ideas al grupo de modo que sean comprensibles y como poco a poco aprenden a escuchar las ideas de los compañeros, incluso cuando no coinciden con su perspectiva. En este contexto, los estudiantes participaron en grupo, al explicar sus ideas y contrastarlas con las de los demás. De este modo, como afirma Araújo (2009), los estudiantes negocian, discuten, escuchan a los demás y respetan sus ideas. Esta es una forma de trabajar los temas políticos y de la democracia en la microsociedad del aula de clase.

Por una parte, las características del diseño de los ambientes de modelación propuestos en esta investigación posibilitó la participación en tanto que promueve que los estudiantes se consideren entre sí como voces fundamentales en la construcción del conocimiento propio y del de los demás. Por otra parte, en cuanto a la participación en los procesos de aprendizaje matemáticos, se hace evidente que se trasciende el contenido concreto y se generan actitudes que pueden variar de una disposición favorable y comprometida hacia el aprendizaje matemático.

Los estudiantes se involucraron en los ambientes de modelación, al desarrollar procesos matemáticos que les permitieron comunicar el proceso y el producto de su actividad y la valoración de la calidad de los procesos, mediante sus actuaciones.

En los dos (2) ambientes de modelación mencionados en este artículo se evidenció como las interacciones y contribuciones permitieron que la participación de los estudiantes se tornara reflexiva y propositiva; ya que ya que no había, en un principio, actitudes de discusión, diálogo y reflexión y al involucrarse en los diferentes procesos fue posible participar en torno a asuntos propios de las matemáticas y a problemáticas sociales en donde las matemáticas son fundamentales para dar respuesta a muchos interrogantes.

Los participantes lograron movilizar su participación al resolver situaciones de su contexto cercano. Lo anterior se evidencia en el incremento de actuaciones como: expresarse en público de manera oral y escrita; compartir y desarrollar el significado matemático con sus compañeros de forma oral y escrita; expresar, discutir y compartir de forma oral o escrita sus ideas y significados matemáticos. La participación en este tipo de ambientes de modelación les permitió a los estudiantes comunicar, discutir y argumentar significados matemáticos en la clase. 
En este artículo, mostramos que existen casos en los cuales la participación, a pesar de sus múltiples manifestaciones y características, se torna en un factor protagónico para propender por los objetivos trazados en la perspectiva socio-crítica de la modelación. En otras palabras, en ambientes de modelación en donde la participación de los estudiantes se torna pasiva y se basa en el seguimiento de instrucciones dadas por los profesores, con dificultad se tendrán experiencias en las cuales los estudiantes se identifiquen como sujetos capaces de producir matemáticas e interpretar y reconocer su rol en la sociedad. Por el contrario, en ambientes de modelación como los descritos en este artículo, las interacciones y las contribuciones promueven formas de participación más activa en las cuales los estudiantes reflexionan, dialogan, toman decisiones y asumen posturas críticas; todo ello coincide con los propósitos de la perspectiva socio-crítica de la modelación.

\section{Agradecimientos}

Aunque no sean responsables por los elementos acá expuestos, agradecemos a los profesores Walter Fernando Castro Gordillo y Jorge Bañol Gutiérrez por la lectura y crítica realizadas a versiones preliminares de este artículo. Agradecemos también a la Fundación Educativa Colegio San Juan Eudes por permitir que la investigación se llevara a cabo con los miembros de su comunidad, y al Comité para el Desarrollo de la Investigación (CODI) de la Universidad de Antioquia por el apoyo financiero al desarrollo de la investigación "la formación inicial de profesores. Aportes de la modelación matemática y las tecnologías digitales" código FPP01, febrero de 2014.

\section{Referencias}

Alves-Mazzotti, Alda y Gewandsznajder, Fernando. (1999). O Método nas ciências naturais e sociais: pesquisa quantitativa e qualitativa. São Paulo: Pioneira.

Araújo, Jussara. (2002). Cálculo, tecnologias e modelagem matemática: as discussões dos alunos. (Tesis de Doctorado, Instituto de Geociências e Ciências Exatas). Universidade Estadual Paulista, Rio Claro, Brasil.

Araújo, Jussara. (2009). Uma abordagem socio-crítica da modelagem matemática: a perspectiva da educação matemática crítica. Alexandria revista de educação em ciências e tecnologia, 2(2), 55-68.

Araújo, Jussara. (2012). Ser crítico em projetos de modelagem em uma perspectiva crítica de educação matemática. Bolema-Boletim de educação matemática, 26(43), 839-860. 
Bahmaei, Farzad. (2013). Mathematical modelling in primary school, advantages and challenges. Journal of mathematical modelling and application, 1(9), 3-13.

Barbosa, Jonei. (2001b). Modelagem matemática e os professores: a questão da formação. Bolema-Boletim de educação matemática, 14(15), 5-23.

Barbosa, Jonei. (2001b). Modelagem matemática e os professores: a questão da formação. Bolema, 15, 5-23.

Barbosa, Jonei. (2003a). What is mathematical modelling?. En Susan Lamon, Willard Parker y S. Ken Houston. (Ed.), Mathematical modelling: a way of life. ICTMA 11 (pp. 227234). England: Horwood Publishing Limited.

Barbosa, Jonei. (2003b). Modelagem matemática na sala de aula. Perspectiva, Erechim (RS), 27(98), 65-74.

Barbosa, Jonei. (julio, 2004). Modelagem Matemática na Sala de Aula. Minicurso presentado en el VIII Encontro Nacional de Educação Matemática realizado en la Universidade Federal de Pernambuco, Recife, Brasil.

Barbosa, Jonei. (2006). Mathematical modelling in classroom: a socio-critical and discursive perspective. ZDM-The international journal of mathematics education, 38(3), 293-301.

Barbosa, Jonei. (2009). Mathematical modelling, the socio-critical perspective and the reflexive discussions. En Morten Blomhøj, S. Carreira. (Eds.), Mathematical applications and modelling in the teaching and learning of mathematics (pp. 133-144). Denmark: Roskilde University.

Biembengut, Maria. (2007). Modelling and applications in primary education. En Werner Blum, Peter Galbraith, Hans-Wolfgang Henn y Mogens Niss (Ed.), Modelling and applications in mathematics education: The 14th ICMI study (pp. 451-456). New York: Springer.

Biembengut, Maria y Hein, Nelson. (2004). Modelación matemática y los desafíos para enseñar matemática. Educación Matemática, 16(2), 105-125.

Blum, Werner, Galbraith, Peter, Henn, Hans-Wolfgang, y Niss, Mogens (Eds.). (2007). Modelling and applications in mathematics education: The 14th ICMI study. New York: Springer.

Bogdan, Robert y Biklen, Sari. (2007). Research for education. An introduction to theories and methods. New York: Pearson.

Civil, Marta, y Planas, Núria. (2004). Participation in the mathematics classroom: does every student have a voice? For the learning of mathematics, 24(1), 7-12.

English, Lyn. (2003). Mathematical modelling with young learners. En Susan Lamon, Willard Parker y S. Ken Houston. (Ed.), Mathematical modelling: a way of life. ICTMA 11 (pp. 19-33). England: Horwood Publishing Limited. 
English, Lyn. (2006). Mathematical modeling in the primary school: children's construction of a consumer guide. Educational studies in mathematics, 63(3), 303-323.

English, Lyn. (2009). Promoting interdisciplinarity through mathematical modelling. ZDM-The international journal of mathematics education, 41(1), 161-181.

English, Lyn. (2010). Mathematical modelling in the primary school. En Ian Putt, Rhonda Faragher, and Mal McLean. (Eds.), Mathematics education for the third millennium: Towards 2010 (pp. 207-214). Australia: James Cook University.

Ferreira, Denise y Wodewotzki, Lúcia. (2007). Modelagem matemática e educação ambiental: uma experiência com alunos do ensino fundamental. Zetetiké, 15(28), 6385.

Gordillo, Mariano. (2006). Conocer, manejar, valorar, participar: los fines de una educación para la ciudadanía. Revista Iberoamericana de Educación, (42), 69-83.

Gorgorió, Núria y Prat, Montserrat. (2009). Jeopardizing learning opportunities in multicultural mathematical classrooms. En Kristiina Kumpulainen, y Margarida César. (Eds.), Social Interactions in Multicultural Settings (pp. 145-170). Rotterdam: Sense Publishers.

Kaiser, Gabriele y Sriraman, Bharath. (2006). A global survey of international perspectives on modelling in mathematics education. ZDM-The international journal of mathematics education, 38(3), 302-310.

Luna, Ana y Alves, Josélia. (2007). Modelagem matemática: as interações discursivas de crianças da $4^{\mathrm{a}}$ série a partir de um estudo sobre anorexia. $V$ Conferência nacional sobre modelagem na educação matemática, 855-876. Universidad Federal de Ouro Preto, Minas Gerais, Brasil.

Malinen, Sanna. (2015). Understanding user participation in online communities: a systematic literature review of empirical studies. Computers in human behavior, 46, 8-38.

Silva, Cíntia y Kato, Lilian. (2012). Quais elementos caracterizam uma atividade de modelagem matemática na perspectiva sociocrítica? Boletim de educação matemática, 26(43), 817-838.

Skovsmose, Ole, Scandiuzzi, Pedro, Valero, Paola y Alrø, Helle. (2011). Aprender matemáticas en posición de frontera: los porvenires y la intencionalidad de los estudiantes en una favela brasilera. Revista Educación y Pedagogía, 23(59), 103-124.

Wenger, Etienne. (1998). Communities of practice: learning, meaning, and identity. New York: Cambridge University Press. 